\title{
Strain variation in ppGpp concentration and RpoS levels in laboratory strains of Escherichia coli K-12
}

Correspondence

Thomas Ferenci

tferenci@mail.usyd.edu.au

Received 13 March 2008

Revised 14 May 2008

Accepted 15 May 2008

\section{Beny Spira, ${ }^{1}$ Xuye $\mathrm{Hu}^{2}$ and Thomas Ferenci ${ }^{2}$}

\author{
${ }^{1}$ Departamento de Microbiologia, Instituto de Ciências Biomédicas, Universidade de São Paulo, São \\ Paulo-SP, Brazil \\ ${ }^{2}$ School of Molecular and Microbial Biosciences, The University of Sydney, NSW 2006, Australia
}

\section{INTRODUCTION}

Large numbers of influential studies in bacterial physiology, genetics and gene regulation have used laboratory strains of Escherichia coli $\mathrm{K}-12$, but evidence is accumulating that stocks kept in various laboratories are not comparable. The MG1655 strain used in genomic studies differs in several respects between laboratories (Soupene et al., 2003). Another commonly used sequenced strain, W3110, also differs in important respects in various stocks (Jishage \& Ishihama, 1997). There are also numerous physiological and regulatory differences between MG1655 and another commonly used K-12 lineage, MC4100 (King et al., 2004). Stored archival cultures of bacteria can be heterogeneous (Sutton et al., 2000), which is also likely to contribute to strain variation in stocks of K-12 strains. We are interested in the molecular basis of strain variation and in this study identify some of the factors important not only in strain differences but also in the settings for global gene regulation in bacteria.

One identified contributor to strain variation is the conditional advantage of the sigma factor $\operatorname{RpoS}\left(\sigma^{\mathrm{S}}\right)$ in

Abbreviation: SPANC, self-protection and nutritional capability. different environments. RpoS is responsible for the general stress response of E. coli (Weber et al., 2005) and strains with high levels of RpoS are more resistant to stress but grow more slowly under a variety of conditions (Ferenci, 2005). The balance between self-protection and nutritional capability, the SPANC balance, is frequently reset by mutation, resulting in many different combinations of stress resistance and nutritional capability in the species (King et al., 2004). Growth of bacteria on poor carbon sources or low levels of nutrients selects for reduced or abolished RpoS levels, whereas stressful environments require RpoS (King et al., 2006). Laboratory strains contain one of several different alleles of rpoS (Atlung et al., 2002). Here we investigate some of the factors that determine RpoS levels and its variation in strains of E. coli K-12.

The role of RpoS in E. coli is complementary to that of the alarmone ppGpp, whose concentration also responds to stress and shifts transcription away from vegetative growth towards stress resistance (Ferenci, 2005; Gentry et al., 1993; Jishage et al., 2002; Typas et al., 2007). ppGpp and RpoS co-operate both mechanistically and strategically, and expression of RpoS-controlled genes is partly dependent on ppGpp (Kvint et al., 2000; Nystrom, 2004). The level of 
ppGpp is also important because it helps to control the amount of RpoS in the cell, as ppGpp increases the cellular concentration of RpoS by several-fold during nutritional stress or upon entry into the stationary phase. The absence of ppGpp impairs or severely delays the accumulation of RpoS (Gentry et al., 1993). ppGpp also positively affects the efficiency of rpoS translation in stationary phase under stress conditions as well as rpoS basal expression under conditions of optimal growth (Brown et al., 2002; Hirsch \& Elliott, 2002). The response to phosphate starvation additionally involves stabilization of RpoS protein mediated by ppGpp (Bougdour \& Gottesman, 2007). At several levels then, ppGpp is intertwined with rpoS regulation and function; here we show ppGpp is also subject to strain variation.

ppGpp directly and indirectly affects many important bacterial characteristics, from stress responses to pathogenesis to biofilm formation (Braeken et al., 2006; Cashel et al., 1996; Magnusson et al., 2005; Nakanishi et al., 2006). ppGpp accumulates in bacteria undergoing nutritional stress, such as amino acid, carbon or phosphate limitation (Bougdour \& Gottesman, 2007; Cashel \& Gallant, 1969; Lazzarini et al., 1971; Spira et al., 1995). Accumulation of ppGpp triggers the 'stringent response', in which a radical decrease in ribosome synthesis results in a general inhibition of protein synthesis and growth arrest (Cashel et al., 1996). The strategic overall role of ppGpp is the allocation of transcriptional resources so they are appropriate to the level of stress and growth rate of the bacteria. This, as with RpoS, necessarily involves a trade-off in the expression of genes related to stress resistance and vegetative growth. The presence of ppGpp may be beneficial in stressful environments but may be an impediment to vegetative growth in intermediate-stress situations. It is therefore interesting to analyse the evolutionary selection pressures imposed on the genes involved in ppGpp synthesis or degradation and how these shape bacterial behaviour.

ppGpp levels are controlled by two proteins, encoded by the genes relA and spot (Cashel et al., 1996). Synthesis of ppGpp under intracellular amino acid starvation is dependent on relA. The RelA protein senses the presence of uncharged tRNAs at ribosomes, which triggers the RelA-dependent synthesis of ppGpp. relA mutants display a relaxed phenotype in response to amino acid limitation, characterized by a further decrease in ppGpp concentration and the continuous production of stable RNA (Cashel et al., 1996). SpoT is a bifunctional cytosolic enzyme capable of both ppGpp synthesis and degradation. Under nutritional stresses other than amino acid limitation, accumulation of ppGpp is largely relA-independent, as the rise in ppGpp concentration is mainly due to inhibition of SpoT hydrolytic activity (Murray \& Bremer, 1996).

Because ppGpp is needed to obtain high levels of $r p o S$ expression, it was expected that ppGpp would also affect the equilibrium between metabolic capacity essential during vegetative growth and stress resistance, the socalled SPANC balance (Ferenci, 2005). ppGpp may have a direct effect on bacterial nutritional ability, as it binds to RNA polymerase and affects the expression of many genes either positively or negatively (Cashel et al., 1996; Magnusson et al., 2005; Reddy et al., 1995). Additionally, ppGpp may affect cellular properties indirectly through RpoS (Cashel et al., 1996); we tested this prediction in this study. We also showed that ppGpp levels are remarkably strain-specific and that spoT is particularly important in microevolution, fixing the basal level of ppGpp in response to selective pressures on stress resistance and nutrition. Finally, we demonstrate that strain variation in ppGpp contributes to the difference in the level of RpoS in E. coli and discuss the consequences of polymorphisms at the core of bacterial regulation.

\section{METHODS}

Strains and media. The strains used in this study are described in Table 1. Bacteria were cultured in T-salts, a Tris-buffered minimal medium supplemented with different concentrations of glucose and $\mathrm{KH}_{2} \mathrm{PO}_{4}$ (Spira et al., 1995), and minimal medium A (MMA; as described by Miller, 1972). For growth on acetate and L-agar plates (Miller, 1972), overnight cultures were harvested, washed twice and resuspended in MMA to a density of $2 \times 10^{8}$ cells $\mathrm{ml}^{-1}$. Aliquots of suspensions $(5 \mu \mathrm{l})$ were spotted on MMA-acetate or L-agar plates and incubated at $37^{\circ} \mathrm{C}$.

Sequence analysis. For the sequencing of the spoT ORF, DNA was amplified by PCR using primers spoTF1 (5'-CAGTATCATGCCCAGTCATTTCTTC-3') and spoTR2 (5'-GGTAGTACTGGTTTCGCCGTGCTC-3'). Sequencing analysis of both DNA strands was performed with primers spoTF1, spoTF2 (5'-AAAAGCGTCGCCGAGCTGGTAGAGG-3'), spoTF3 (5'-TGATCGGCCCGCACGGTGTGCCGG-3'), spoTF5 (5'-TGATCGGCCCGCACGGTGTGCCGG-3'), spoTR1 (5'-TGCACCATCGCCATAATCATCTTGC-3'), spoTR2 and spoTR3 (5'-CTTGATTTCGGTGATGAACTCCTG-3') at the Australian Genome Research Facility. Sequences were assembled with the help of the Staden Package (http://staden.sourceforge.net/).

Transductional transfer of spoT alleles and rpoS::Km. The different spoT alleles were transferred by P1 transduction (Miller, 1972). Strains CF12269, CF12271 and CF12273 served as lysate donors for the spoT1655, spoT202 and spoT203 alleles, respectively, and MC4100TF served as the recipient strain. The proximity of the spot locus to zib563:: $\operatorname{Tn} 10$ (80\% co-transduction) was used to select the transductants for tetracycline resistance; thes transductants were later tested for spoT-dependent phenotypes, such as ppGpp level, iodine staining and growth on acetate. For the transduction of the spoT4100 allele into MG1655, the zib563:: Tn 10 marker was first transduced into MC4100 and then a lysate of MC4100 zib563::Tn10 was used to transduce MG1655. The rpoS:: Km mutation was transduced into other strains using strain ZK1000 as a donor.

ppGpp assay. ppGpp was extracted from cells growing at $37{ }^{\circ} \mathrm{C}$ in Tsalts minimal medium containing $100 \mu \mathrm{Ci}(3.7 \mathrm{MBq}) \mathrm{KH}_{2}{ }^{32} \mathrm{PO}_{4} \mathrm{ml}^{-1}$. To measure basal levels of ppGpp, cells growing exponentially in T-salts were resuspended in $\mathrm{T}$-salts supplemented with $0.2 \%$ glucose and $0.2 \mathrm{mM} \mathrm{KH}_{2}{ }^{32} \mathrm{PO}_{4}$ at $\mathrm{OD}_{600}$ 0.1. Samples were harvested after 90, 100, 110 or 120 min. ppGpp was extracted from amino acid-starved cells 
Table 1. E. coli strains used in this study

\begin{tabular}{|c|c|c|}
\hline Strain & Relevant genotype & Reference/source \\
\hline CF12269 & N99 relA1 zib563:: Tn10 spot $T^{+}($spoT1655) & M. Cashel \\
\hline CF12271 & N99 relA1 zib563:: Tn10 spoT202 & M. Cashel \\
\hline CF1693 & MG1655 $\Delta$ relA:: Km & M. Cashel \\
\hline MC4100 & $\mathrm{F}^{-} \operatorname{araD139}(\operatorname{argF-lac}) \mathrm{U} 169 \mathrm{rpsL150}$ deoC1 relA1 thiA ptsF25 flbB5301 rbsR & Lab collection \\
\hline MC4100BS & MC4100 from B. Spira lab & Lab collection \\
\hline MC4100TF relA & MC4100TF $\Delta$ relA:: Km (transduction from CF1693) & This study \\
\hline MC4100TF rpos & MC4100TF rpoS:: Km (transduction from ZK1000) & This study \\
\hline MC4100TF spoT1655 & MC4100TF spoT1655 zib563:: Tn10 (transduction from CF12269) & This study \\
\hline MC4100TF spoT202 & MC4100TF spoT202 zib563::Tn10 (transduction from CF12271) & This study \\
\hline MC4100TF spoT202 rpos & $\begin{array}{l}\text { MC4100TF spoT202 rpoS:: Km zib563:: Tn10 (transduction from CF12271 } \\
\text { and ZK1000) }\end{array}$ & This study \\
\hline MG1655 & Wild-type E. coli K-12 & Lab collection \\
\hline MG1655 relA & MG1655 $\Delta$ relA:: Km (transduction from CF1693) & This study \\
\hline MG1655 rpos & MG1655 rpoS:: Km (transduction from ZK1000) & This study \\
\hline MG1655 spoT4100BS & MG1655 spoT4100BS (spoT from MC4100BS) zib563:: Tn 10 & This study \\
\hline MG1655 spoT4100BS rpoS & MG1655 spoT4100BS zib563:: Tn10 rpoS:: Tn10 & This study \\
\hline MG1655 spoT4100TF & MG1655 spoT4100TF (spoT from MC4100TF) zib563:: Tn10 & This study \\
\hline MG1655 spoT4100TF rpos & MG1655 spoT4100TF zib563:: Tn10 rpoS:: Tn10 & This study \\
\hline ZK1000 & ZK126 rpoS:: Km & Bohannon et al. (1991) \\
\hline
\end{tabular}

using the same method except that amino acid starvation was triggered by adding $1 \mathrm{mg}$ serine hydroxamate $\mathrm{ml}^{-1}$ to the cultures.

The labelled samples were mixed immediately with 0.5 vols of cold formic acid and stored overnight at $-20{ }^{\circ} \mathrm{C}$. The extracts were centrifuged for $5 \mathrm{~min}$ at 10000 r.p.m. to precipitate cell debris; 3 $5 \mu \mathrm{l}$ was applied to PEI-cellulose TLC plates. The labelled nucleotides were resolved by one-dimensional TLC using $1.5 \mathrm{M}$ $\mathrm{KH}_{2} \mathrm{PO}_{4}$ as solvent. The amounts of ppGpp on the chromatograms were estimated by measuring the radioactivity of the spots in a Phosphor-Imager (Molecular Dynamics) and calculating the level of ppGpp relative to that of GTP + ppGpp. The densitometric analysis was performed with the help of the Image $J$ software (available at http://rsb.info.nih.gov/ij/).

Determination of growth rate. The growth rate of K-12 strains was measured in cultures growing in unlimited T-salts minimal medium. Overnight cultures were diluted to $\mathrm{OD}_{600} 0.02$ and grown at $37^{\circ} \mathrm{C}$ for $4 \mathrm{~h}$. Samples were taken every hour and $\mathrm{OD}_{600}$ was determined. The growth rate $\left(\mu, \mathrm{h}^{-1}\right)$ was calculated using the formula $\mu=\ln \left(N / N_{0}\right) / t$, where $N_{0}$ is the initial and $N$ is the final cell concentration, and $t$ is the time course of the growth curve.

Steady-state growth conditions in chemostats. T-salts supplemented with $0.02 \%$ glucose and $1.0 \mathrm{mM} \mathrm{KH}_{2} \mathrm{PO}_{4}$ was used to set up an $80 \mathrm{ml}$ chemostat culture as described by Notley-McRobb et al. (2002). The dilution rate was set to $0.1 \mathrm{~h}^{-1}$. Daily samples were taken to monitor the rpoS status of members of the population. The rpoS status was determined by iodine staining (see below).

Detection of rpoS status by iodine staining. The RpoS level was qualitatively assessed by staining glycogen with an iodine solution as described by Hengge-Aronis \& Fischer (1992). Patches of bacteria or diluted chemostat samples were grown overnight on L-agar plates, stored at $4{ }^{\circ} \mathrm{C}$ for $24 \mathrm{~h}$ and then flooded with iodine. The intensity of the brown colour varies according to glycogen concentration in the cell and indirectly measures the level of RpoS (King et al., 2004; Spira \& Ferenci, 2008). $r p o S^{+}$strains stain brown to dark brown.

Stress resistance assays. Bacteria from overnight cultures grown in L-broth were washed twice in $0.9 \%(\mathrm{w} / \mathrm{v}) \mathrm{NaCl}$ and diluted in $0.9 \% \mathrm{NaCl}$ to a density of $4 \times 10^{3}$ cells $\mathrm{ml}^{-1}$. For oxidative stress, freshly diluted $\mathrm{H}_{2} \mathrm{O}_{2}$ (final concentration $1.5 \mathrm{mM}$ ) was added to $1 \mathrm{ml}$ culture and held at room temperature. Samples $(100 \mu \mathrm{l})$ were taken at 5, 10, 15 and $20 \mathrm{~min}$, spread on L-agar plates and incubated overnight at $37^{\circ} \mathrm{C}$. For osmolarity, suspensions of $4 \times 10^{3}$ cells $\mathrm{ml}^{-1}$ were incubated in $1.5 \mathrm{M} \mathrm{NaCl}$ at room temperature. Samples $(100 \mu \mathrm{l})$ were withdrawn at 1,3 and $4 \mathrm{~h}$ and plated onto L-agar. For cold stress, bacteria resuspended in $0.9 \% \mathrm{NaCl}$ were held at $4{ }^{\circ} \mathrm{C}$ and survival was assessed at 2, 4, 6 and 8 days.

Quantification of Rpos. Bacteria from overnight cultures were resuspended in L-broth and grown at $37{ }^{\circ} \mathrm{C}$ up to $\mathrm{OD}_{600} 2.0$, then $2 \times 10^{9}$ cells were centrifuged, resuspended in $200 \mu \mathrm{l}$ application buffer $(0.5 \mathrm{M}$ Tris/HCl, $2 \%$ SDS, $5 \%$ 2-mercaptoethanol, $10 \%$, v/v, glycerol and $0.01 \%$ bromophenol blue) and boiled for $5 \mathrm{~min}$. Proteins were resolved in a $12.5 \%$ denaturing polyacrylamide gel and transferred to a nitrocelullose membrane (GE HealthCare) by capillary action. Following blocking with $5 \%$ skim milk, the membrane was incubated with 2000 -fold diluted monoclonal antiRpoS antibodies (Neoclone) and 20000-fold diluted peroxidaseconjugated anti-mouse IgG (Pierce). The Super Signal West Pico kit (Pierce) was used to detect the RpoS bands as recommended by the manufacturer. The membrane was exposed to X-ray films for various periods of time and the signal intensities were scanned and computed using the Image J software. 


\section{RESULTS}

\section{Level of ppGpp and nature of the relA/spoT genes in a high-Rpos strain}

The MC4100 strain from the Ferenci lab (subsequently called MC4100TF) maintains higher levels of RpoS than other K-12 strains, including MG1655 (King et al., 2004). As ppGpp is an important factor in controlling RpoS synthesis and function (Gentry et al., 1993; Kvint et al., 2000), the level of ppGpp in MC4100 was compared to that of MG1655. As shown in Table 2, the basal level of ppGpp was twofold greater in MC4100TF than in MG1655 under steady-state growth conditions at similar growth rates. The higher ppGpp in MC4100 was unexpected, given the known genotypes $\left(\mathrm{relA}^{+}\right.$in MG1655 and relA1 in MC4100) and the wild-type spoT genes in both. Amino acid starvation induced by an amino acid analogue resulted in a relaxed response in MC4100TF consistent with the presence of the relA1 defect (data not shown), which was closer to the expected result. To test if undetected genetic differences were present, both the relA and spoT genes associated with ppGpp synthesis were sequenced in MC4100TF. The relA1 insertion mutation in MC4100 was indeed found in MC4100TF (data not shown). Surprisingly, the spoT sequence in MC4100TF differed from that in MG1655 and included a substitution (H255Y)

Table 2. ppGpp basal levels in different genetic backgrounds

ppGpp was extracted from exponential-phase cells growing in T-salts. Values represent the radioactive spots of ppGpp/GTP + ppGpp and are the mean $( \pm S D)$ of at least three independent experiments.

\begin{tabular}{|c|c|c|}
\hline Strain/genotype & ppGpp & Growth rate* \\
\hline MC4100BS & $0.27( \pm 0.02)$ & 0.67 \\
\hline \multicolumn{3}{|l|}{ MC4100TF } \\
\hline MC4100TF & $0.31( \pm 0.03)$ & 0.53 \\
\hline rpos & $0.28( \pm 0.02)$ & 0.65 \\
\hline$\Delta r e l A$ & $0.29( \pm 0.03)$ & 0.54 \\
\hline spot202 & $0.42( \pm 0.01)$ & 0.31 \\
\hline spoT202 rpos & $0.4( \pm 0.02)$ & 0.45 \\
\hline spot203 & $0.39( \pm 0.02)$ & $0.68 \dagger$ \\
\hline spoT203 rpos & $0.43( \pm 0.01)$ & 0.46 \\
\hline spoT1655 & $0.12( \pm 0.01)$ & 0.62 \\
\hline \multicolumn{3}{|l|}{ MG1655 } \\
\hline MG1655 & $0.15( \pm 0.01)$ & 0.49 \\
\hline rpos & $0.15( \pm 0.02)$ & 0.53 \\
\hline$\Delta r e l A$ & $0.15( \pm 0.02)$ & 0.53 \\
\hline spoT4100 & $0.33( \pm 0.01)$ & 0.45 \\
\hline spoT4100 rpos & $0.3( \pm 0.0)$ & 0.44 \\
\hline spoT4100BS & $0.27( \pm 0.01)$ & 0.44 \\
\hline spoT4100BS rpos & $0.37( \pm 0.01)$ & 0.44 \\
\hline
\end{tabular}

*Values represent the growth rates $\left(\mu, \mathrm{h}^{-1}\right)$ of exponentially growing cells in T-salts minimal medium.

$\dagger$ Growth rate is unusually high due to the appearance of fast-growing mutants (see Fig. 2). and a two-amino acid insertion between residues 82 and 83 (+QD) of the SpoT protein. The gene with this pair of changes is subsequently called spoT4100.

To test if ppGpp differences occurred as a result of the MC4100TF spoT mutation, the spoT4100 gene of MC4100TF was transferred into MG1655 by co-transduction with a linked marker. Reciprocally, the wild-type spoT gene of MG1655 was transferred to MC4100TF. The identity of the spoT allele in all transductants in Table 2 was confirmed by DNA sequencing. As shown in Table 2, the effect of the spoT4100 gene on MG1655 was to increase the basal level of ppGpp. Consistent with this, introduction of the wild-type spoT from MG1655 into MC4100TF reduced the ppGpp level to that found in MG1655. Hence the spoT4100 allele is likely to be responsible for the elevated basal level of ppGpp in MC4100TF. However, the spoT4100 gene does not result in a rise in ppGpp to the extent found with the previously isolated spoT202 and spoT203 mutations (Sarubbi et al., 1988) when transferred to the same background (Table 2).

A different MC4100 stock maintained in another laboratory, MC4100BS, was also sequenced for spoT and contained the same pair of changes as MC4100TF. The spoT4100 from MC4100BS also elevated ppGpp in the MG1655 background to similar basal levels as found in the MC4100TF transductant. The mutational differences in spoT and ppGpp between MG1655 and MC4100 indicate the variability of these features of E. coli laboratory strains.

\section{Differences in RpoS levels between MG1655 and MC4100 lineages}

The effect of different endogenous ppGpp concentrations on the level of RpoS protein were tested. As shown in Fig. 1, using anti-RpoS antibodies and three to six replicate immunoblots of each strain, different levels of RpoS were found both as a function of strain background and as a result of different spoT alleles. The difference between MG1655 and MC4100TF (Fig. 1) is fully consistent with an earlier study (King et al., 2004). More surprisingly, in spoT1655 strains, RpoS levels were also lower in MG1655 than in MC4100TF. Since both MC4100TF transduced with the spoT1655 allele and MG1655 showed similar ppGpp levels (Table 2), it is necessary to invoke other differences in RpoS regulation to explain the protein levels. The increased RpoS levels found in spoT4100 strains relative to spoT1655 strains were consistently higher in either MC4100TF or MG1655 backgrounds. Likewise, ppGpp was approximately twofold higher in the spoT4100 strain. With the spoT202/203 mutations and even higher ppGpp, RpoS did not increase substantially above the spoT4100 level, suggesting saturation of rpoS regulation by ppGpp. The trends in protein amount in Fig. 1 between spoT1655 and spoT mutants are consistent with the effect of ppGpp levels (Table 2) on rpoS expression (Gentry et al., 1993). 


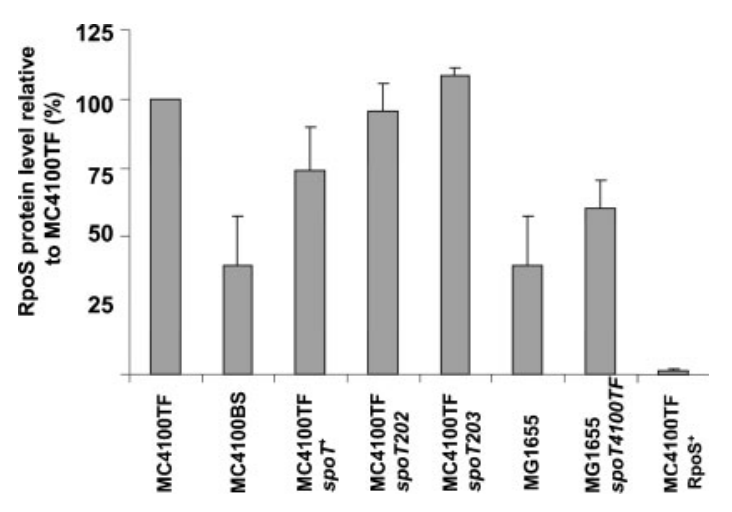

Fig. 1. Quantification of RpoS protein. Bacteria were harvested from early stationary phase cultures of strains with various spoT alleles; samples were lysed and proteins were resolved by SDSPAGE. The blots were incubated with anti-RpoS monoclonal antibodies. The bands were scanned and quantified. Values represent the mean \pm SEM of at least three independent experiments. A mutant lacking RpoS is also shown.

A more unexpected result was that the RpoS level in MC4100BS was considerably lower than that in MC4100TF. Given that the two MC4100 stocks have the same ppGpp level and the same spoT allele, and that MC4100TF and MC4100BS also have the same rpoS sequence (unpublished results), it is necessary to postulate that there is also a strain difference in another of the numerous extragenic inputs into the control of RpoS synthesis or degradation (Hengge-Aronis, 2002). These MC4100 stocks had a common ancestor over 30 years ago (Casadaban, 1976), but have been in separate laboratories for more than 25 years. Independent propagation or storage conditions have led to strain variation in RpoS of one or both strains due to mutations outside spoT. Sequencing of the MC4100TF chromosome that is currently under way may help to resolve the additional causes of high RpoS in this strain.

\section{Effect of ppGpp and spoT differences on growth rates, nutrition and the SPANC balance}

The influence of spoT alleles on RpoS levels suggested that ppGpp would also affect downstream RpoS-dependent phenotypes. A simple test of RpoS function is through testing for glycogen accumulation, which is RpoS-dependent and proportional to its protein level (Spira \& Ferenci, 2008). As shown in Fig. 2(c), staining with iodine showed that glycogen accumulation is very much in parallel with the independently determined RpoS estimations. The strong staining in the high-ppGpp, high-RpoS strains (MC4100TF spoT202, MC4100TF spoT203) contrasts with the poor staining in MG1655 and MC4100BS. It is also obvious that there is an increase in staining in spoT4100 compared with spoT1655. These data are entirely consistent with those in Fig. 1. (a)

Acetate growth

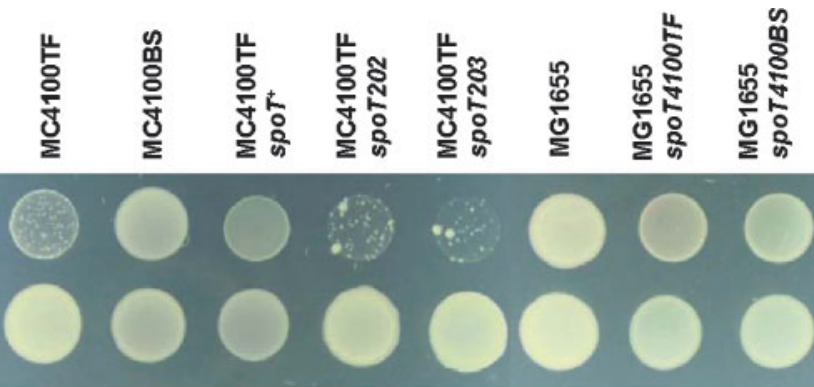

(b) L-agar Growth

(c) lodine staining
RpoS $^{+}$

Rpos:

$\mathbf{R p o S}^{+}$

Rpos:

$\mathbf{R p o S}^{+}$

Rpos
Fig. 2. Growth of bacterial patches on different carbon sources. Washed cultures $(5 \mu \mathrm{l})$ containing equal amounts of cells were grown on minimal agar plates containing $0.4 \%$ acetate as the sole carbon source (a); the faster-growing colonies in some of the slowgrowing patches are rpoS mutants selected after 2 days on plates (King et al., 2004). Bacterial patches were grown on L-agar as described above and growth was recorded after $12 \mathrm{~h}$ at $37{ }^{\circ} \mathrm{C}(\mathrm{b})$; these patches were stained with iodine to detect the level of glycogen in the cell (c). The intensity of staining is a function of glycogen content and indirectly of RpoS protein concentration (Spira \& Ferenci, 2008). 
The level of RpoS determines the nutritional competence of $E$. coli cultured on poor carbon sources; $E$ coli displays weak growth on substrates such as acetate when RpoS levels are high, as in MC4100TF (King et al., 2004). The effect of different spoT alleles was tested on the growth on acetate (Fig. 2a). The low-ppGpp, low-RpoS strain, MG1655, grows much better on acetate than MC4100TF. Elevation of ppGpp in MG1655 slows growth somewhat, but not as much as in MC4100TF. The slower growth on acetate is corrected by an rpoS null mutation, and indeed rpoS mutants are seen as faster-growing colonies within the MC4100TF patch (King et al., 2004). An rpoS mutation does not affect ppGpp levels (Table 1), so the growth reduction that is seen on acetate is more likely to result from the indirect influence of ppGpp on RpoS levels. Consistent with this notion, artificially increasing ppGpp levels through the spoT4100 allele or the characterized spoT202 or spoT203 alleles (Sarubbi et al., 1988) also reduced growth on acetate. The growth defect could be relieved by introduction of rpos mutations into these strains (Fig. 2).

The difference in growth rates with the set of strains in Table 2 was evident, even with good carbon sources such as glucose. Furthermore, growth rate differences can be seen even in rich Luria-Bertani medium (Fig. 2b). The slower growth of high-ppGpp strains was at least partially due to RpoS, as rpoS mutants were able to grow faster even with rich nutrients. Even with the very high-ppGpp strains containing the spoT202/203 alleles, an rpoS mutation relieves most of the growth inhibition. Hence, selection for improved growth rates with any carbon source can be a potential cause of polymorphisms affecting RpoS and ppGpp.

\section{Effect of ppGpp differences on stress resistance}

The spot alleles affected the nutritional side of the SPANC balance, so twofold variation in basal ppGpp levels, such as between MC4100TF and MC4100TF spoT1655, should also be reflected in differences in the general stress responses of the bacteria. The effects of $\mathrm{H}_{2} \mathrm{O}_{2}$, high osmolarity and cold stress were tested on these strains (Fig. 3). In each stress condition, the strain with the spoT4100 allele performed better. Particularly interesting is the disparity upon cold storage. Given the advantage of the spoT4100 allele in stressful situations (as may occur in prolonged laboratory storage at $4{ }^{\circ} \mathrm{C}$ ), the difference in stress survival may explain the selection pressure for mutations in spoT towards higher ppGpp levels in the MC4100 lineage or any E. coli strain stored for long periods in a stressful environment. The above results suggest that an increase in basal ppGpp levels can improve resistance and tilt the SPANC balance away from effective nutrition; lower ppGpp improves growth on different carbon sources, but reduces stress survival.

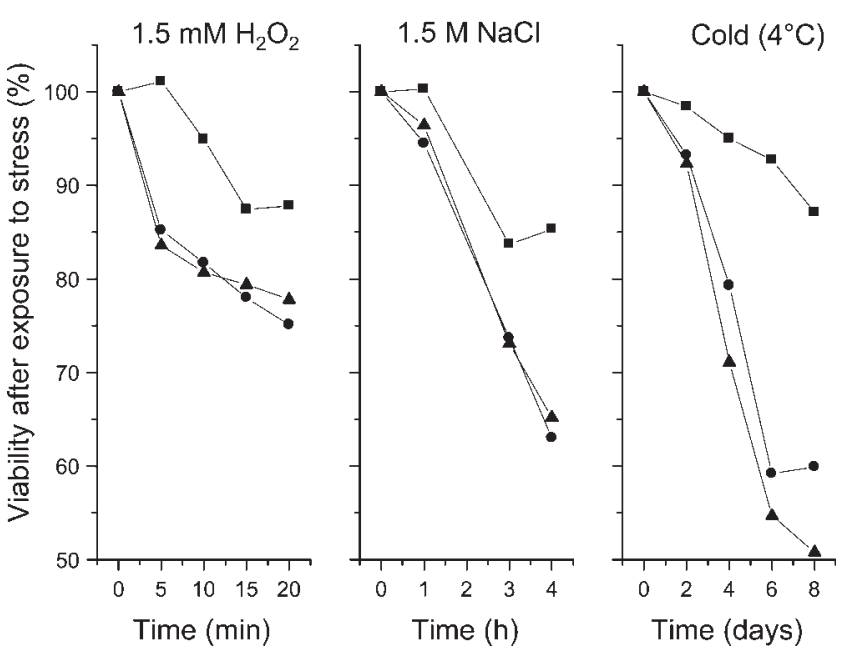

Fig. 3. Effect of the spoT background on stress resistance. Cultures of strains MC4100TF ( $\mathbf{\square})$, MC4100TF spoT1655 (A) and MC4100BS (O) were challenged for resistance to $1.5 \mathrm{mM}$ $\mathrm{H}_{2} \mathrm{O}_{2}$, high osmolarity $(1.5 \mathrm{M} \mathrm{NaCl})$ and cold $\left(4{ }^{\circ} \mathrm{C}\right)$. Viable bacteria were determined from plate counts.

\section{Connection between variations in ppGpp levels and the enrichment of rpoS mutations in $E$. coli populations}

Previous results indicated that the high level of RpoS in strains such as MC4100TF is associated with a measurably greater incidence of rpoS mutations in nutrient-limited populations than that which is found with low-RpoS strains like MG1655 (King et al., 2004). To see if the elevated ppGpp resulting from spoT4100 influenced the selection pressure for rpoS mutations under nutrient limitation, the kinetics of spread of rpoS mutations was followed in chemostat cultures limited by glucose, with all cultures growing at the same rate $\left(\mu=0.1 \mathrm{~h}^{-1}\right)$. The rate of enrichment of rpoS mutations was measured by determining the decrease in $\mathrm{RpoS}^{+}$cells in strains containing different spoT alleles as shown in Fig. 4. The populations containing spoT alleles resulting in higher ppGpp levels (spoT4100, spoT202) started to accumulate significant numbers of rpoS mutations within 2 days of continuous culture. The spoT202 mutant with the highest ppGpp is particularly prone to RpoS change; more than $50 \%$ of the population were RpoS ${ }^{-}$after $24 \mathrm{~h}$ of culture. When the wild-type spoT gene (MC4100TF spoT1655), rpoS mutations still proliferated in the culture, but more slowly. MG1655 did not accumulate mutations in rpoS under these conditions. Introduction of spoT4100 into MG1655 increased the selective pressure on rpoS, resulting in a measurable rate of accumulation of rpoS mutations. This assay measures the benefit of the loss of rpoS (King et al., 2006), so it can be concluded that the nature of spoT influences the burden imposed by RpoS under nutrient limitation. Interestingly, pairs of strains containing the same spoT allele (MG1655 and MC4100TF spoT1655; or 


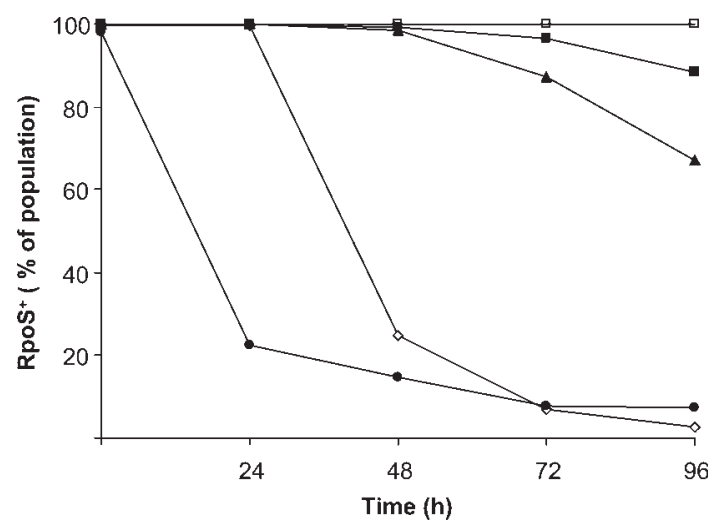

Fig. 4. Loss of RpoS in bacteria with different ppGpp backgrounds during steady-state growth. Cultures of strains MC4100TF $(\diamond)$, MC4100TF spoT1655 (A), MC4100TF spoT202 (•), MG1655 ( $\square$ ) and MG1655 spoT4100 (ם) were grown separately for 4 days in glucose-limited chemostats at a $0.1 \mathrm{~h}^{-1}$ dilution rate. Samples were harvested daily, diluted and plated on L-agar. Changes in rpoS status were followed by iodine staining. The proportion of $\mathrm{RpoS}^{+}$cells is shown; the value for each strain is representative of at least two experiments.

MG1655 spoT4100 and MC4100TF) had similar ppGpp levels (Table 2) but exhibited different rates of rpoS mutant accumulation. Hence, differences between the MC4100 and MG1655 backgrounds besides ppGpp need to be invoked to explain the extent of rpoS changes under glucose limitation.

\section{DISCUSSION}

The spoT gene is central to the ppGpp-dependent response of bacteria to changes in growth rate and nutrient starvation (Cashel et al., 1996) so it is sobering to note that it is subject to polymorphism, as the sequence variation in laboratory strains of $E$. coli suggests. It is probably no historical accident that the spoT variations were first noted in some laboratory lineages that differed from other K-12 strains and exhibited a 'spotless' phenotype (Laffler \& Gallant, 1974). Distinct mutations in spoT were also found in E. coli after thousands of generations of laboratory growth on glucose (Cooper et al., 2003), suggesting that spoT is subject to selection under repeat-batch culture conditions as well.

The structure-function relationships in the bi-functional SpoT protein are only partly understood (Gentry \& Cashel, 1996), so the spoT mutations found in this study can only be tentatively interpreted. The insertion at amino acid residues 82/84 probably compromises the ppGppase activity of the protein. Interestingly, one of the highppGpp mutations in spoT (spoT202) is nearby, affecting residue 78 (T78I, M. Cashel, personal communication). The spoT203 mutation affecting residue 140 (R140C, M.
Cashel, personal communication) and the H255Y substitution in the spoT4100 strain are more likely to affect synthesis in a positive way. Further protein-function studies are needed to fully resolve these questions, as current speculations are based on the structure of a hybrid protein related to SpoT (Hogg et al., 2004).

In many respects, spoT4100 resembles the previously described spoT1 mutation that had elevated basal levels of ppGpp (Lagosky \& Chang, 1980; Sarubbi et al., 1989). Strain variation in spoT has already been associated with the presence of a spoT1 allele in a subset of K-12 lineages (Laffler \& Gallant, 1974). Indeed, sequencing of the archival spoT1 gene revealed the same double mutation as in MC4100 (M. Cashel, personal communication), so it is possible that an undocumented spoT1 mutation was incorporated into the MC4100 strain during its complex construction (Casadaban, 1976). A full genealogical analysis of the studied strains was not undertaken, however, as it is clear from the strain variation, even between the two MC4100 stocks (the TF and BS variants), that frequent changes are common in stored cultures. It is also unclear whether spoT1-like mutations are more prevalent in relA1 mutants.

The environmental influence on ppGpp regulation is affected by the same dichotomy already observed and discussed for RpoS (Ferenci, 2005), namely the different needs of the cell in response to nutrient limitation and stress resistance. As described for the MC4100TF strain and its spoT1655 derivative, or MG1655 and its spoT4100 derivative, the nature of the spoT allele that is present in $E$. coli simultaneously influences the level of RpoS, stress resistance and nutritional capabilities of E. coli. The benefit of high basal ppGpp levels is seen in the greater stress resistance that is conferred (Fig. 3), whereas the benefit of low ppGpp is evident from increased growth, particularly under nutrient limitation or with poor nutrients (Fig. 2a, b). Depending on the environment, the antagonistic pleiotropy between resistance and nutrition can change the cell's SPANC balance. Given that external stresses and nutritional constraints are common in natural settings, it is not surprising that mutations occur and are constantly selected in shifting environments. Indeed, the variation in spoT resembles the polymorphisms in rpos, which are, if anything, even more extensive (Atlung et al., 2002; Ferenci, 2005).

In the past, the relationship between growth rate and ppGpp levels was discussed mainly in the context of high ppGpp resulting in a reduction in stable RNA synthesis and thereby reduced translational capacity (Sarubbi et al., 1988). After the effect of ppGpp on RpoS was discovered (Gentry et al., 1993), it was suggested that some of the ppGpp effect could be indirect and could be acting through elevated expression of the alternative sigma factor (Cashel et al., 1996). Indeed, as shown in this study, the elimination of RpoS results in faster growth on a range of substrates. Even on rich media, the inhibitory effect of artificially 
elevated ppGpp can be largely overcome by rpoS mutations. Hence, the growth rate effects of high ppGpp are more complex than simple control of translational processes and they probably involve competition between sigma factors as well. Adding to the complexity, both ppGpp and RpoS levels differ between strains, so the overall effect of elevated ppGpp is also likely to be strainspecific.

In summary, the physiological and regulatory roles of RpoS and ppGpp are interlinked at several levels, including strain variation. Increased ppGpp generally has a positive effect on RpoS levels, but differences in RpoS between the MC4100 and MG1655 backgrounds demonstrate that other factors besides ppGpp need to be invoked to explain the extent of rpoS changes. Previous results have pointed to considerable polymorphisms in rpoS between E. coli strains (Ferenci, 2003); these new results suggest that at least one of the genes involved in ppGpp synthesis and degradation is also subject to the same kind of selective pressures as is rpoS. In this respect, spoT and rpoS are involved in the dynamic balancing of SPANC within a bacterium in response to the nature of the immediate environment and demand for nutrients. These findings are significant for molecular studies of bacteria, because it is now known that two of the global systems that control the allocation of transcriptional resources are actually strain-specific, since ppGpp and RpoS levels are potentially non-uniform in $E$. coli under identical growth conditions. This has major consequences for global studies of the pattern of expression of hundreds of genes controlled directly or indirectly (by competition) at the level of RNA polymerase. Equally importantly, many of the numerous traits controlled by ppGpp (Braeken et al., 2006; Cashel et al., 1996; Magnusson et al., 2005; Nakanishi et al., 2006) are also likely to be subject to strain variation.

\section{ACKNOWLEDGEMENTS}

This study was greatly supported by contributions from M. Cashel, who supplied strains, helpful comments and unpublished information on spoT mutants. This work was supported by Fundação de Amparo a Pesquisa do Estado de São Paulo (FAPESP-Brazil) and an Australian Endeavour Research Fellowship (to B.S.), as well as the Australian Research Council (to T. F.).

\section{REFERENCES}

Atlung, T., Nielsen, H. V. \& Hansen, F. G. (2002). Characterisation of the allelic variation in the rpoS gene in thirteen $\mathrm{K}-12$ and six other non-pathogenic Escherichia coli strains. Mol Genet Genomics 266, 873881.

Bohannon, D. E., Connell, N., Keener, J., Tormo, A., Espinosa-Urgel, M., Zambrano, M. M. \& Kolter, R. (1991). Stationary-phase-inducible "gearbox" promoters: differential effects of katF mutations and role of sigma 70. J Bacteriol 173, 4482-4492.

Bougdour, A. \& Gottesman, S. (2007). ppGpp regulation of RpoS degradation via anti-adaptor protein IraP. Proc Natl Acad Sci U S A 104, 12896-12901.
Braeken, K., Moris, M., Daniels, R., Vanderleyden, J. \& Michiels, J. (2006). New horizons for (p)ppGpp in bacterial and plant physiology. Trends Microbiol 14, 45-54.

Brown, L., Gentry, D., Elliott, T. \& Cashel, M. (2002). DksA affects ppGpp induction of RpoS at a translational level. J Bacteriol 184, 4455-4465.

Casadaban, M. J. (1976). Transposition and fusion of the lac genes to selected promoters in Escherichia coli using bacteriophage Lambda and Mu. J Mol Biol 104, 541-555.

Cashel, M. \& Gallant, J. (1969). Two compounds implicated in the function of the RC gene of Escherichia coli. Nature 221, 838-841.

Cashel, M., Gentry, D., Hernandez, V. J. \& Vinella, D. (1996). The stringent response. In Escherichia coli and Salmonella: Cellular and Molecular Biology, pp. 1458-1496. Edited by F. C. Neidhardt, R. Curtiss III, J. L. Ingraham, E. C. C. Lin, K. B. Low, B. Magasanik, W. S. Reznikoff, M. Riley, M. Schaechter \& H. E. Umbarger. Washington, DC: American Society for Microbiology.

Cooper, T. F., Rozen, D. E. \& Lenski, R. E. (2003). Parallel changes in gene expression after 20,000 generations of evolution in Escherichia coli. Proc Natl Acad Sci U S A 100, 1072-1077.

Ferenci, T. (2003). What is driving the acquisition of mutS and rpoS polymorphisms in Escherichia coli? Trends Microbiol 11, 457-461.

Ferenci, T. (2005). Maintaining a healthy SPANC balance through regulatory and mutational adaptation. Mol Microbiol 57, 1-8.

Gentry, D. R. \& Cashel, M. (1996). Mutational analysis of the Escherichia coli spoT gene identifies distinct but overlapping regions involved in ppGpp synthesis and degradation. Mol Microbiol 19, 1373-1384.

Gentry, D. R., Hernadez, V. J., Nguyen, L. H., Jensen, D. B. \& Cashel, M. (1993). Synthesis of stationary-phase sigma factor sigma-S is positively regulated by ppGpp. J Bacteriol 175, 7982-7989.

Hengge-Aronis, R. (2002). Signal transduction and regulatory mechanisms involved in control of the sigma(S) (RpoS) subunit of RNA polymerase. Microbiol Mol Biol Rev 66, 373-395.

Hengge-Aronis, R. \& Fischer, D. (1992). Identification and molecular analysis of $g l g S$, a novel growth-phase-regulated and rpoS-dependent gene involved in glycogen synthesis in Escherichia coli. Mol Microbiol 6, 1877-1886.

Hirsch, M. \& Elliott, T. (2002). Role of ppGpp in rpoS stationary-phase regulation in Escherichia coli. J Bacteriol 184, 5077-5087.

Hogg, T., Mechold, U., Malke, H., Cashel, M. \& Hilgenfeld, R. (2004). Conformational antagonism between opposing active sites in a bifunctional RelA/SpoT homolog modulates (p)ppGpp metabolism during the stringent response. Cell 117, 57-68.

Jishage, M. \& Ishihama, A. (1997). Variation in RNA polymerase sigma subunit composition within different stocks of Escherichia coli W3110. J Bacteriol 179, 959-963.

Jishage, M., Kvint, K., Shingler, V. \& Nystrom, T. (2002). Regulation of sigma factor competition by the alarmone ppGpp. Genes Dev 16, $1260-1270$.

King, T., Ishihama, A., Kori, A. \& Ferenci, T. (2004). A regulatory trade-off as a source of strain variation in the species Escherichia coli. $J$ Bacteriol 186, 5614-5620.

King, T., Seeto, S. \& Ferenci, T. (2006). Genotype-by-environment interactions influencing the emergence of rpos mutations in Eschetichia coli populations. Genetics 172, 2071-2079.

Kvint, K., Farewell, A. \& Nystrom, T. (2000). RpoS-dependent promoters require guanosine tetraphosphate for induction even in the presence of high levels of sigma(s). J Biol Chem 275, 14795-14798.

Laffler, T. \& Gallant, J. (1974). spoT, a new genetic locus involved in stringent response in E. coli. Cell 1, 27-30. 
Lagosky, P. A. \& Chang, F. N. (1980). Influence of amino-acid starvation on guanosine $5^{\prime}$-diphosphate $3^{\prime}$-diphosphate basal-level synthesis in Escherichia coli. J Bacteriol 144, 499-508.

Lazzarini, R. A., Cashel, M. \& Gallant, J. (1971). On the regulation of guanosine tetraphosphate levels in stringent and relaxed strains of Escherichia coli. J Biol Chem 246, 4381-4385.

Magnusson, L. U., Farewell, A. \& Nystrom, T. (2005). ppGpp: a global regulator in Escherichia coli. Trends Microbiol 13, 236-242.

Miller, J. (1972). Experiments in Molecular Genetics. Cold Spring Harbor, NY: Cold Spring Harbor Laboratory.

Murray, K. D. \& Bremer, H. (1996). Control of SpoT-dependent ppGpp synthesis and degradation in Escherichia coli. J Mol Biol 259, 41-57.

Nakanishi, N., Abe, H., Ogura, Y., Hayashi, T., Tashiro, K., Kuhara, S., Sugimoto, N. \& Tobe, T. (2006). ppGpp with DksA controls gene expression in the locus of enterocyte effacement (LEE) pathogenicity island of enterohaemorrhagic Escherichia coli through activation of two virulence regulatory genes. Mol Microbiol 61, 194-205.

Notley-McRobb, L., King, T. \& Ferenci, T. (2002). rpoS mutations and loss of general stress resistance in Escherichia coli populations as a consequence of conflict between competing stress responses. J Bacteriol 184, 806-811.

Nystrom, T. (2004). Growth versus maintenance: a trade-off dictated by RNA polymerase availability and sigma factor competition? Mol Microbiol 54, 855-862.

Reddy, P. S., Raghavan, A. \& Chatterji, D. (1995). Evidence for a ppGppbinding site on Escherichia coli RNA polymerase: proximity relationship with the rifampicin-binding domain. Mol Microbiol 15, 255-265.

Sarubbi, E., Rudd, K. E. \& Cashel, M. (1988). Basal ppGpp level adjustment shown by new spoT mutants affect steady state growth rates and $r r n A$ ribosomal promoter regulation in Escherichia coli. Mol Gen Genet 213, 214-222.

Sarubbi, E., Rudd, K. E., Xiao, H., Ikehara, K., Kalman, M. \& Cashel, M. (1989). Characterization of the spoT gene of Escherichia coli. J Biol Chem 264, 15074-15082.

Soupene, E., van Heeswijk, W. C., Plumbridge, J., Stewart, V., Bertenthal, D., Lee, H., Prasad, G., Paliy, O., Charernnoppakul, P. \& Kustu, S. (2003). Physiological studies of Escherichia coli strain MG1655: growth defects and apparent cross-regulation of gene expression. J Bacteriol 185, 5611-5626.

Spira, B. \& Ferenci, T. (2008). Alkaline phosphatase as a reporter of $\sigma^{\mathrm{S}}$ levels and rpoS polymorphisms in different E. coli strains. Arch Microbiol 189, 43-47.

Spira, B., Silberstein, N. \& Yagil, E. (1995). Guanosine $3^{\prime}, 5^{\prime}-$ bispyrophosphate (ppGpp) synthesis in cells of Escherichia coli starved for Pi. J Bacteriol 177, 4053-4058.

Sutton, A., Buencamino, R. \& Eisenstark, A. (2000). rpoS mutants in archival cultures of Salmonella enterica serovar Typhimurium. J Bacteriol 182, 4375-4379.

Typas, A., Becker, G. \& Hengge, R. (2007). The molecular basis of selective promoter activation by the sigma(S) subunit of RNA polymerase. Mol Microbiol 63, 1296-1306.

Weber, H., Polen, T., Heuveling, J., Wendisch, V. F. \& Hengge, R. (2005). Genome-wide analysis of the general stress response network in Escherichia coli: sigma(S)-dependent genes, promoters, and sigma factor selectivity. J Bacteriol 187, 1591-1603.

Edited by: J. Green 